\title{
DECOMPOSITION OF THE SYMPTOM OBSERVATION MATRIX AND GREY FORECASTING IN VIBRATION CONDITION MONITORING OF MACHINES
}

\author{
CZESŁAW CEMPEL \\ Institute of Applied Mechanics \\ Poznań University of Technology, ul. Piotrowo 3, 60-965 Poznań, Poland \\ e-mail: Czeslaw.cempel@put.poznan.pl
}

\begin{abstract}
With the tools of modern metrology we can measure almost all variables in the phenomenon field of a working machine, and many of the measured quantities can be symptoms of machine conditions. On this basis, we can form a symptom observation matrix (SOM) intended for condition monitoring and wear trend (fault) identification. On the other hand, we know that contemporary complex machines may have many modes of failure, called faults. The paper presents a method of the extraction of the information about faults from the symptom observation matrix by means of singular value decomposition (SVD), in the form of generalized fault symptoms. As the readings of the symptoms can be unstable, the moving average of the SOM is applied with success. An attempt to assess the diagnostic contribution of a primary symptom is made, and also an approach to assess the symptom limit value and to connect the SVD methodology with neural nets is considered. Finally, a condition forecasting problem is discussed and an application of grey system theory (GST) to symptom prognosis is presented. These possibilities are illustrated by processing data taken directly from the machine vibration condition monitoring area.
\end{abstract}

Keywords: machine wear, multidimensional observation, vibration, SVD decomposition, fault space, observation space, symptom limit value, forecasting, grey system theory.

\section{Introduction}

The idea of multidimensional diagnostics of machines using the symptom observation matrix and the application of the singular value decomposition were proposed some years ago (Cempel 1999; Cempel et al., 2007). It enables us to extract the information on the developing machine faults using the component symptom observation vector $m$. By successive discrete readings (observations) of this vector at time moments $n \Delta \theta$ in a machine lifetime $\theta$, one can create an $m \times n(n>m)$ perpendicular (non-square) observation matrix called the symptom observation matrix (SOM). This is our only source of information on evolving faults (wear trends) in the running machine. The application of singular value decomposition (SVD) to this set of diagnostic data enables us to observe the evolution of a few generalized faults of the diagnosed machine, starting from the fault of maximal severity. Applying next the concept of symptom reliability (Cempel et al. 2000; Natke et al. 1997; Cempel, 1991) to the so extracted generalized fault symptoms, one can calculate the symptom limit va- lue $S_{l}$, the basis for any diagnostic decision. However, the loadings of machines by production processes (or the environment) are not constant, so that the resulting symptom readings may have some disturbances influencing the assessment of the machine condition. This disturbing influence is most important at the start-up of a new machine, because, as usual in machine diagnostics, we normalize symptoms to starting (initial) healthy values.

One of the possibilities of reducing these errors is to rescale the current symptom reading to a standard load if such a load assessment is possible. This can be done by measurements of some quantities connected with a production process, with the wind load, or the sea waving, depending on the nature of disturbances. In (Cempel et al., 2007), it was shown that the idea of symptom rescaling is workable, giving the possibility of a better assessment of the machine condition working in a nonstationary loading regime. But, when the assessment of the load parameter is not possible, we can use with success the averaging of a few starting symptom readings, as was shown in (Cempel et al., 2007). These promising results regarding reducing 
operational instabilities and random disturbances of observed symptoms lead us towards the employment of SVD to the averaged SOM applied to the whole matrix, and in this approach the last trial of averaging encompasses the few starting values of the SOM (Cempel et al., 2006). There is another possible approach to treat the multidimensionality of observations, i.e., by means of principal component analysis (PCA) (Korbicz et al., 2004; Pantopian et al., 1999). However, this approach tends to disregard smaller damage information, due to the inherent squaring effect of singular values in PCA.

Having such tools, it seems reasonable to look for the analysis and optimization of the whole procedure of diagnostic information extraction from the SOM. This possibility may give us some additional insight and hints as well as allow us to form the research goals on the road to automated machine diagnostics. Finally, the aim of machine diagnostics is to assess the future machine condition and the residual lifetime. For this purpose, for the first time we will apply grey system theory, a very promising tool developed by Deng (1989).

This is the aim of our paper, and we will verify these concepts taking into account real cases of machine condition monitoring with operational instabilities, random disturbances of readings, and, of course, normal stationary running conditions.

\section{Multidimensional observation of conditions and the extraction of fault symptoms}

Having in mind the above, consider a critical machine in operation. During its working life $0<\theta<\theta_{b}\left(\theta_{b}\right.$ stands for the anticipated breakdown time), several independent faults $F_{t}(\theta), t=1,2, \ldots, u$ evolve and grow as some wear processes, leading to the destruction of the machine. Hence, we would like to identify and assess the advancement of these faults by forming and measuring the symptom observation vector $\left[S_{m}\right]=\left[S_{1}, \ldots, S_{r}\right]$, which may have components physically different, like vibration amplitudes, temperature, machine load, lifetime $\theta$, etc.

In order to track the machine condition (the evolution of faults) by these measurements, we make equidistant readings of the above symptom observation vector at the lifetime moments $\theta_{n}, n=1, \ldots, p, \quad \theta_{p} \leq \theta_{b}$, forming in this way the rows of the SOM. From previous research (Cempel, 1999; Cempel et al., 2006) we know that the best way of SOM preprocessing is to centre it (subtract), and normalize it (divide) to the symptom initial value $S_{m}(0)=S_{0 m}, m=1, \ldots, r$ of each given symptom (a column of the SOM). It is also known from that research that the amount of diagnostic information in the SOM increases if we append the lifetime $\theta$ column as the first approximation of the system logistic vector $L$ and the load (Natke et al., 2002; Cempel, 2005). Finally, we will also apply a three-point moving average procedure to the successful symptom readings, as was shown in (Cempel et al., 2006).

After such preprocessing, we will obtain a dimensionless SOM in the form

$$
S O M \equiv O_{p r}=\left[S_{n m}\right], \quad S_{n m}=\frac{\mathbf{S}_{n m}}{\mathbf{S}_{0 m}}-1,
$$

where boldface nonitalic letters indicate primary measured and averaged dimensional symptoms.

As was already said in the introduction, we apply now SVD (Golub et al., 1983; Kiełbasiński et al., 1992) to the dimensionless SOM (1) to obtain singular components of the SOM in the form

$$
O_{p r}=U_{p p} \Sigma_{p r} V_{r r}^{T}
$$

where $U_{p p}$ is a $p \times p$ orthonormal matrix of left singular vectors, $V_{r r}$ is an $r \times r$ orthonormal matrix of right singular vectors, and the diagonal matrix of singular values $\Sigma_{p r}$ is defined as

$$
\Sigma_{p r}=\operatorname{diag}\left(\sigma_{1}, \ldots, \sigma_{l}\right),
$$

where $\sigma_{1}>\sigma_{2}>\cdots>\sigma_{u}>0$, and $\sigma_{u+1}=\cdots=\sigma_{l}=$ $0, l=\max (p, r), u \leq \min (p, r), u<r<p$.

Finally it can be shown that every perpendicular matrix has such a decomposition (2), and it may be also interpreted as the product of three matrices (Will, 2005), namely,

$$
O_{p r}=(\text { Hanger })(\text { Stretcher })\left(\text { Aligner }^{T}\right) .
$$

This is a very metaphorical description of the SVD transformation, but it seems to be a useful analogy for the inference and decision making in our case. In terms of condition monitoring, the above decomposition means that from $r$ primarily measured symptoms (the dimension of the observation space) we can extract only $u \leq r$ independent sources of diagnostic information, describing evolving generalized faults $F_{t}$, creating in this way the fault space (see Fig. 1). As can be seen in the upper right panel of Fig. 1, only a few developing faults make essential contributions to the total fault information. The rest of generalized faults are below the standard $10 \%$ noise level. What is important here is that such SVD decomposition can be made on-line, after each new observation (reading) of the symptom vector $\left[S_{m}\right], n=1, \ldots, p$, and in this way we can trace the fault evolution (wear processes) in an operating mechanical system.

Based on the current research and implementation of this idea (Cempel, 2003), we can say that the most important fault oriented indices obtained from SVD are the first pair $\left(S D_{t}, \sigma_{t}\right), t=1,2$, and also the pair of the sums of all indices, $S u m S D_{i}$. The first fault indices $S D_{t}$ can be named as the discriminant or the generalized symptom of 

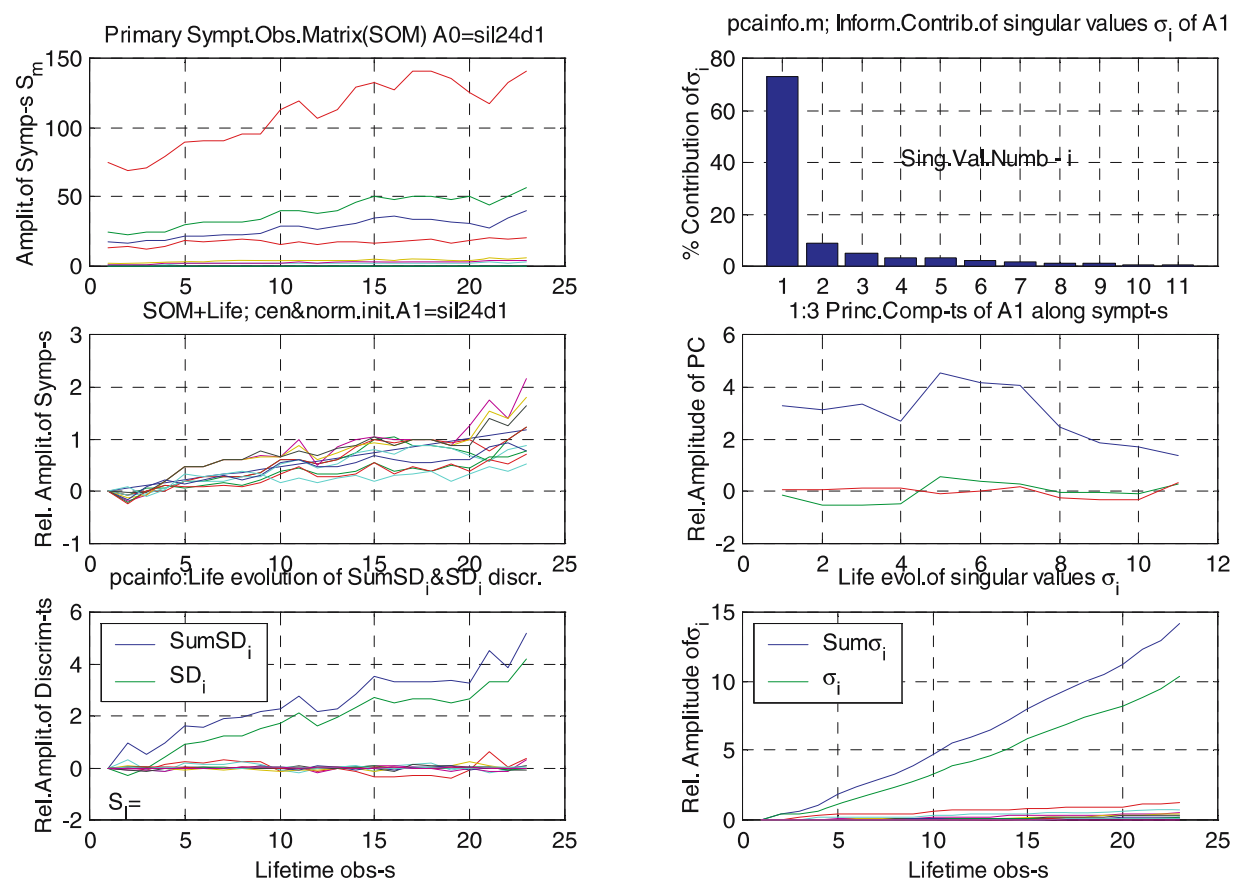

Fig. 1. SOM of the railway diesel engine vibration, processed by an early diagnostic program pcainfo.m based on SVD

the fault $t$, and one can get it as the SOM product and the singular vector $v_{t}$ as follows:

$$
S D_{t}=O_{p r} v_{t}=\sigma_{t} u_{t}, \quad t=1, \ldots, u .
$$

From SVD theory we know (Golub et al., 1983; Kiełbasiński et al., 1992) that all singular vectors $v_{t}, u_{t}$ are orthonormal (orthogonal and normalized to one), so the energy norm of this new discriminant is simply

$$
\operatorname{Norm}\left(S D_{t}\right)=\left\|S D_{t}\right\|=\sigma_{t}, \quad t=1, \ldots, u .
$$

The above discriminant $S D_{t}(\theta)$ can be also named the lifetime fault profile and, in turn, the singular value $\sigma_{t}(\theta)$ as a function of the lifetime seems to be its advancement (the energy norm).

Similar fault inference can be postulated for the meaning and evolution of summation quantities, which can mean the total damage profile $\operatorname{Sum} S D_{i}(\theta)$ and the total damage advancement $\operatorname{Sum}_{i}(\theta)$ as follows:

$$
\begin{aligned}
\operatorname{SumS}_{i}(\theta) & =\sum_{i=1}^{z} S D_{i}(\theta)=\sum_{i=1}^{z} \sigma_{i}(\theta) u_{i}(\theta), \\
\operatorname{Sum}_{i}(\theta) & =\sum_{i=1}^{z} \sigma_{i}(\theta) \sim \sum_{i=1}^{z} F(\theta)_{i} .
\end{aligned}
$$

The proportionality sign in the above relation indicates a possible way of inference. But the meaning of that relation with $\Sigma \sigma_{i}(\theta)$ seems not to be fully validated experimentally. Generally, it seems that the condition inference based on the above summation measures $\Sigma\left(S D_{i}\right)$ may stand for the first approach to multidimensional condition inference, as was clearly shown in previous papers (Cempel et al., 2006a; Cempel et al., 2006b; Cempel, 2005; Żółtowski et al., 2004).

Having in mind the optimization of the observation space, one additional remark should be made concerning the SOM. It will be good to measure in some way the information content of the SOM. This can be done by calculating the Frobenius norm (Frob) of this matrix, and the volume $(\mathrm{Vol})$ created by this $u$-dimensional fault space obtained by the application of singular value decomposition. We can calculate the former as the square root of the sum of squares of singular values (Kiełbasiński, 1992), and the latter as the product of non-squared singular values $\sigma_{i}$. However, we know that the last singular values are very small quantities and the squaring operation will reduce the value of our measure, making it insensitive to small contributions of such information. Hence, we will define both measures as the sum and product of singular values only,

$$
\operatorname{Frob1}(S O M)=\left(\Sigma \sigma_{i}\right), \operatorname{Vol} 1(S O M)=\Pi \sigma_{i},
$$

$i=1, \ldots, u$.

Looking for the values of this measure and the number of nonzero essential singular values $\sigma_{i}$, we may have some assessment of the importance of the fault space obtained by the SVD transformation of the SOM. But how to assess the relative importance of primary measured symptoms as a component of the observation vector $\left[S_{m}\right]$ ? If we examine the left-hand side of (4) and the role of right 
singular vectors $\nu_{t}$, we can come to the conclusion that the components of $\nu_{t}$ are the weights of primary symptoms in the creation of generalized fault symptoms. This can be seen better when we write down explicitly the first, and diagnostically most important, generalized fault symptom

$$
S D_{1}(\theta)=\Sigma S_{i}(\theta) v_{i 1} .
$$

This means that the weights $\nu_{i 1}$ can be treated as normalized sensitivity measures of primary symptoms in the creation of generalized fault symptoms. What is more, using the analogy of neural nets (NNs) (Dunham 2003, p. 62) we can treat the generalized fault $S D_{t}(\theta)$ as the output of a singular neuron, and again the components of Vector $\nu_{i 1}$ can be treated as the input weights, or the sensitivity weights, of our primary symptoms. We will use this useful analogy later on.

Finally, let us return to the diagnostic interpretation of the very useful formula (2a). Using its left-hand side we are stretching the SOM over the life (observations) dimension, obtaining the matrix of generalized symptoms $S D$. Using its right-hand side, we are stretching the SOM over the observation dimension, obtaining the contribution matrix $A L$, assessing in this way the contribution of each primary symptom to the generalized fault symptom $S D_{i}$,

$$
\begin{aligned}
& S D=O_{p r} V_{r r}=U_{p p} \Sigma_{r r}, \\
& A L=U_{p p}^{T} O_{p r}=\Sigma_{r r} V_{r r}^{T} .
\end{aligned}
$$

We will calculate the above matrices and use them for a better interpretation of monitoring results $(S D)$, and the optimization of the dimension of the observation space $(A L)$.

\section{Examples of simple and advanced SVD decomposition of real diagnostic cases}

We have explained all essential steps and transformations on the way from the measured symptom space to the required fault space. Let us illustrate these steps and assess their diagnostic value on data taken from real cases of machinery condition monitoring. Let us begin our step-bystep trip with the illustration of the influence of preprocessing of the SOM, shown using the example of diesel engine vibration condition monitoring data processed by the program pcainfo.m 1 , as in Figs. 1 and 2. Here nine vibration amplitude symptoms were measured (average, rms, peak amplitudes of vibration acceleration, velocity and displacement), every $10,000 \mathrm{~km}$ distance of the railway diesel engine, on the top of its 12 th cylinder. Our diagnostic measurement starts from the repair time of the engine and lasts up to its overhaul at $230 \mathrm{~km}$.

\footnotetext{
${ }^{1}$ This Matlab version of a principal component procedure analysis is based on SVD, so the concept of a principal component of PCA and a singular vector of $S V D$ can be used equivalently here.
}

The upper-left panel of Fig. 1 presents the life course of the primary SOM with a dominant symptom, namely, the peak vibration acceleration amplitude at the top and average displacement amplitude symptom at the bottom. Applying any decomposition program to such an unbalanced data set in Fig. 1, PCA or SVD will make the principal component very similar to the dominant symptom, as was shown in (Cempel, 1999). Consequently, the middle-left panel shows the transformed SOM (appended also with the straight line of the system lifetime $\theta$ as the additional symptom). This means it is centred and normalized to the initial value of each component of the symptom vector. This operation on the SOM brings us a divergent bundle of symptom life curves (middle-left panel), with the range and different dynamics of evolution only. Now, after preprocessing, it has the same range from 0 up to 2, approximately. Even now, as we can see in the bottom-left panel presenting the evolution of lifetime generalized faults, there is only one dominant generalized fault $S D_{1}$, and the summation generalized symptom $\operatorname{SumSD}(\theta)$ does not differ significantly. The same can be seen in the upper and bottom left panels, where the singular values $\sigma_{i}$ normalized to their sums and their lifetime evolution are shown. Now one can be sure that there is only one dominant mode of damage in the running diesel engine described by our symptom space (defined by the SOM), and by using the extraction power of SVD it was possible to capture the evolution of this fault. The contribution of primary symptoms of the transformed SOM to the creation of generalized faults is shown in the middle-right panel. One can see here that the contributions of Symptoms 9-11 to the creation of the symptom SD1 is the lowest one, and in the optimization attempt of the observation space they can be omitted in a first approach.

Drawing computational conclusions from the program just presented and its results, one can see that the lifetime evolution of singular values $\sigma_{t}(\theta)$ does not bring much new diagnostic information. Also, it will be much better to see instead the real dimensionless contribution of primary symptoms, presenting these in terms of dominant components of $U$ (the hanger matrix) and $V$ (the aligner matrix) shown by (2a). In order to be sure that the addition of the system lifetime as a new symptom and the SOM preprocessing are favorable, two forms of the SOM are processed in a new version of the program svdneur.m, one without a preprocessed primary SOM and the second with a transformed one. The transformed SOM includes the lifetime symptom addition, centring, normalization to the initial value, and three-point moving averaging (which changes the initial value mentioned). Let us see the diagnostic inference power of the new program without SOM preprocessing through the window of the same engine data, as is shown in Fig. 2. The organization of Fig. 2 is similar to the previous one, with the addition of three hanger and aligner matrix components. We can see 

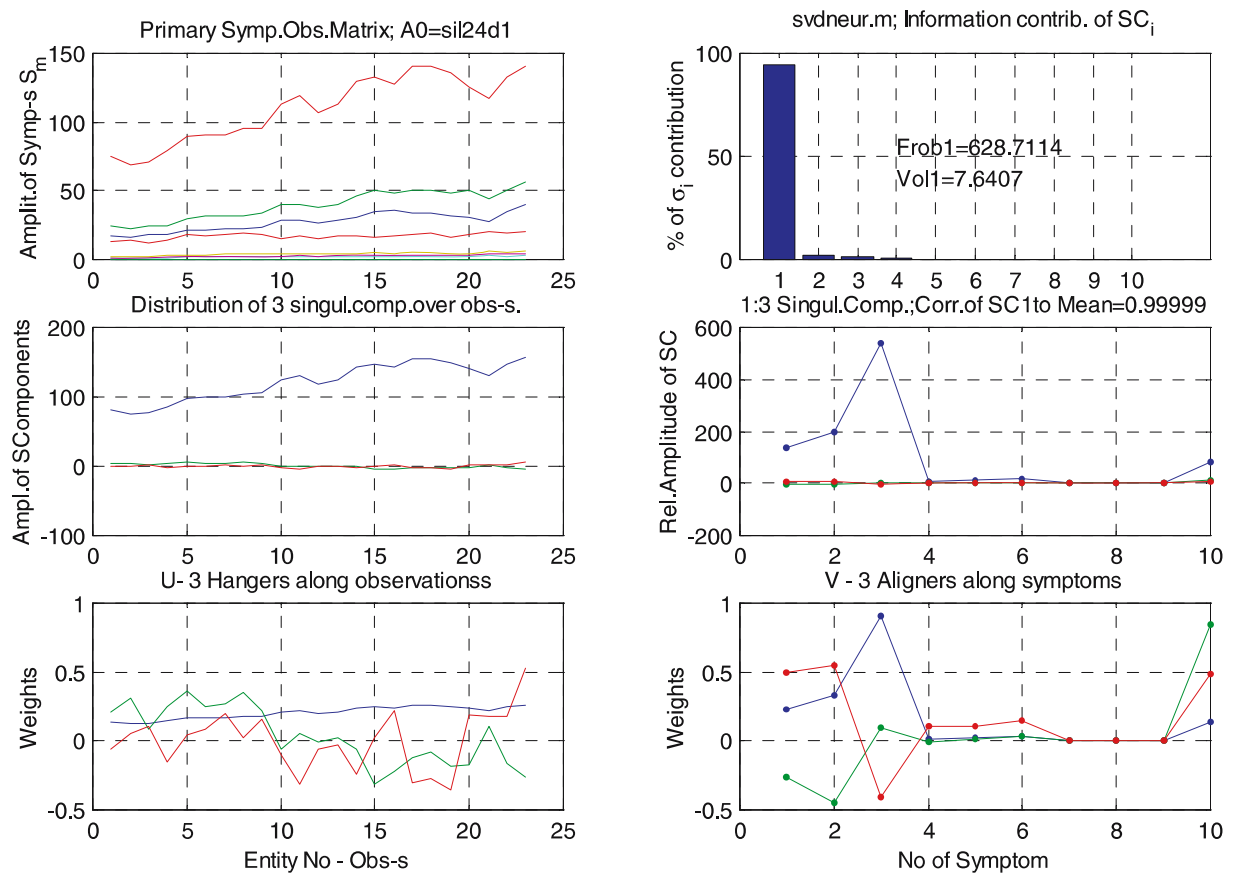

Fig. 2. Primary SOM of the same diesel engine processed by a new program svdneur. $m$ with new possibilities of assessing the symptom contribution of hanger and aligner matrices.

here that the dominance of the peak vibration acceleration symptom (top left) can be observable in all subplots, being the first generalized fault symptom (middle-left panel), and its influence (Symptom 3 in the SOM) can only be comparable to the other acceleration Symptoms 1 and 2. The maximal gain from the transformation of the SOM can be noticed from the comparison of the middle panels of Figs. 1 and 2. There is no doubt that all symptoms must have the same range, and using the symptom of a different physical origin in some cases of condition monitoring, we use a transformation of the SOM as in Fig. 1 in each case.

The running conditions of machines, such as load or environmental interactions, can be hard to control given some stochastic components at every symptom reading. This was the reason why we have previously introduced the three-point moving average of each component of the observation vector. Next figures will show how it works in a real measurement situation.

As the next example, let us analyze multi-symptom observations of bearings tested on an accelerated wear stand. Here seven vibroacoustic symptoms were measured together with the power of the driving motor and the temperature of the bearing outer race. In each case of the new bearing test, the power of the driving motor fell down abruptly as a result of bearing driving. Moreover, this phenomenon disturbs greatly the observation and the resulting processing of the SOM. But the moving average operation of the SOM columns can remove this inconvenience totally, as can be seen when comparing Figs. 3 and 4 .
The running-in symptom values were totally cancelled (Fig. 4) when the moving average operation was applied to the same data. Also the extraction and differentiation of the generalized fault symptom SD1 (the middleleft panel) is obvious. The same concerns the contribution of primary symptoms in the creation of SD1 (the middle left panel), where Symptoms 7 and 8 (driving power and race temperature) can be neglected without loss of information.

Note the decreasing Frobenius norm of the SOM and the volume of the fault space due to transformation. At the same time, the rank of the SOM is not changed. Also note that the contribution of singular values $\sigma_{i}$ was changed much (upper-right panel) due to removing the influence of the receding symptom.

Finally, one can say that the evolution of the generalized fault SD1 obtained now, as a result of the processing of the transformed SOM, is almost monotonic. In this way, it is just ready to undertake the go/do not go diagnostic decision at the end of the bearing diagnostic test. This can be done by an operator or automatically, when applying the concept and calculation of symptom reliability and the symptom limit value $S_{l}$ (Cempel et al. 2000; Natke, 2002; Cempel 1991).

From the ball bearing test stand, let us pass to the huge fan with a rotor mass of three tons, which pumps air into the mining shaft, where the demand for the air is uncontrollable. As is shown in Fig. 5, even the transformed SOM gives unstable symptoms. But the SVD operation 

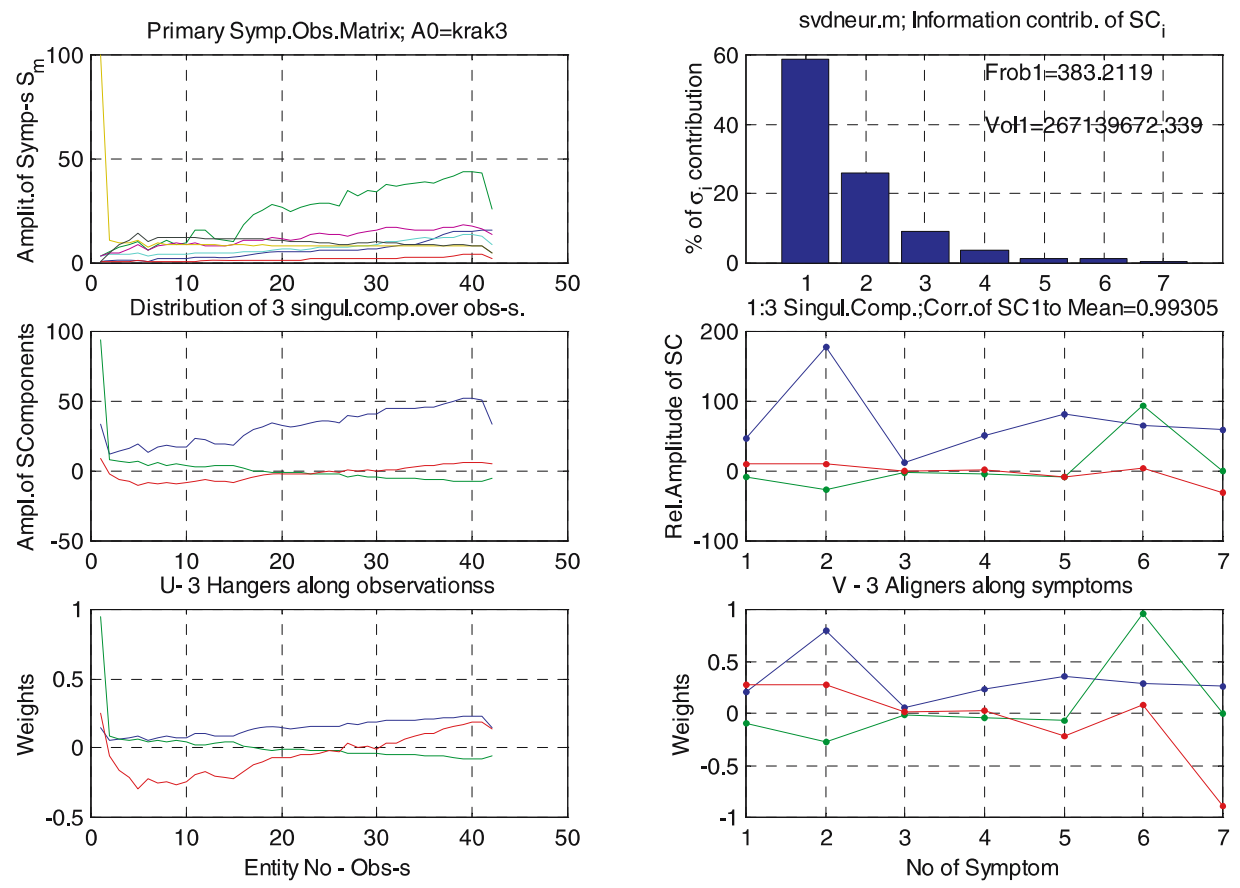

Fig. 3. Multisymptom diagnostic observation of the rolling bearing krak3 on an accelerated test stand with an untransformed SOM and a visible bearing driving for one period.
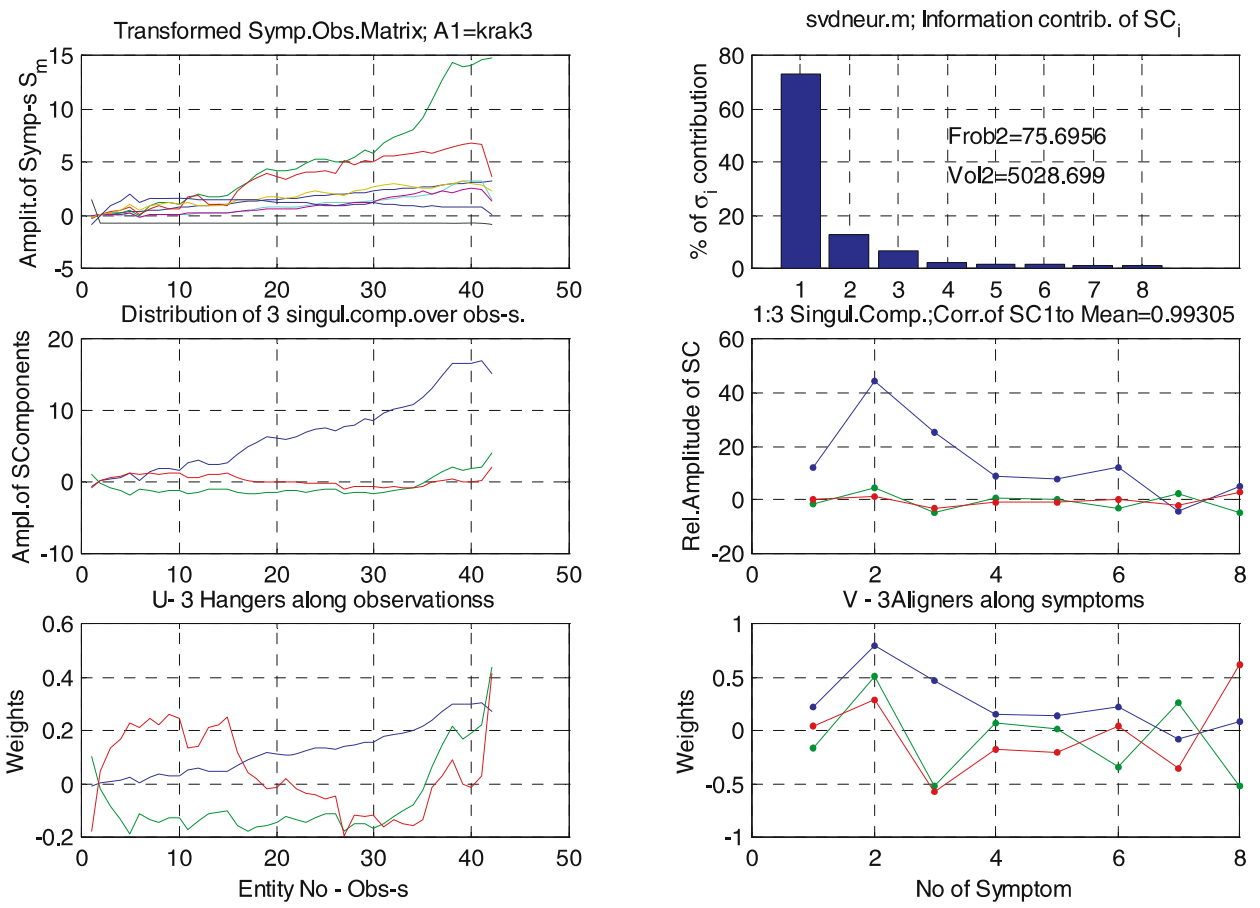

Fig. 4. Bearing test stand as before krak3, but with processing using a transformed SOM. 

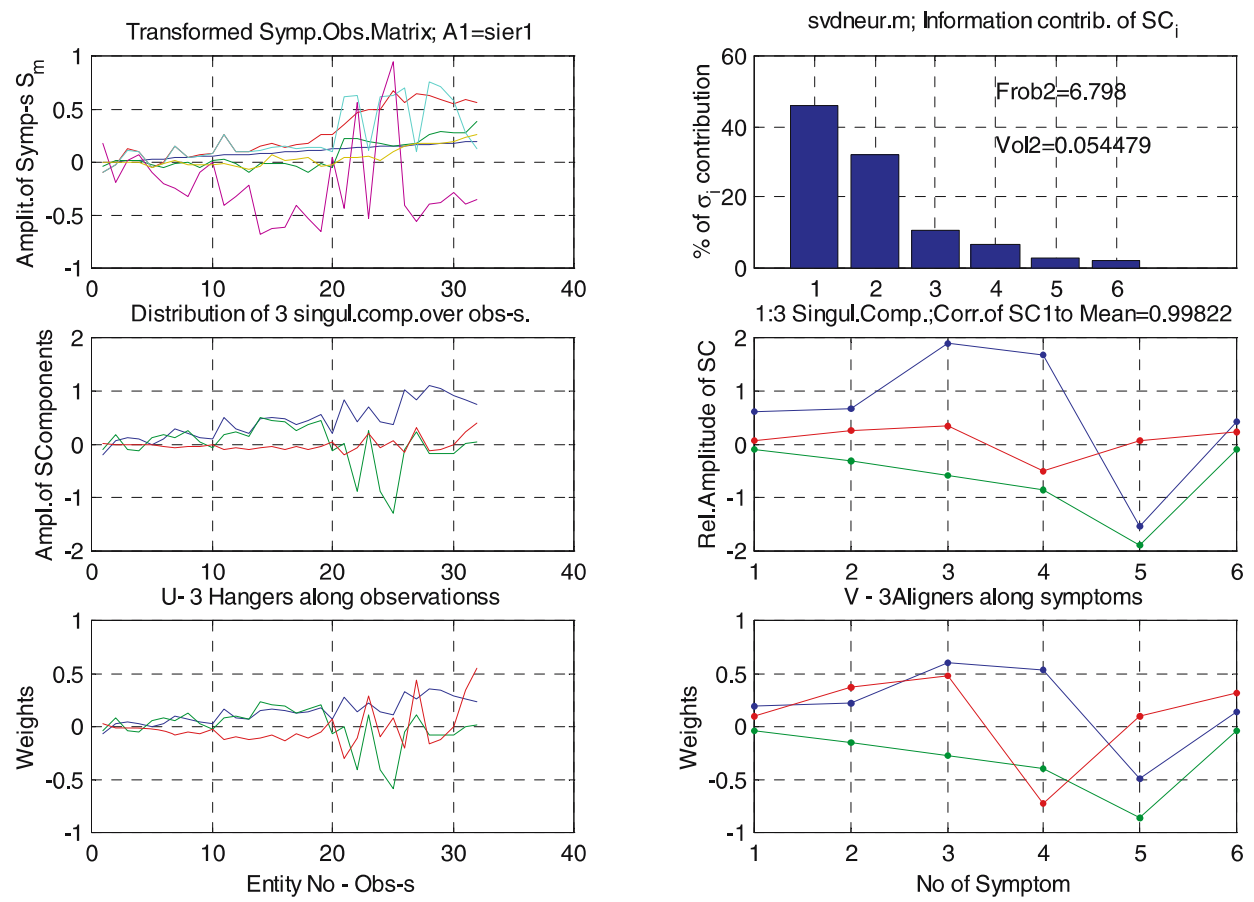

Fig. 5. Vibration diagnostics of a huge fan for the ventilation of mining shaft, where the air demand (load) is uncontrollable, but possible to diagnoze with the program svdneur.m.

on the SOM allows us to distinguish a prevailing generalized fault SD1, and another one which is unstable. When we apply a rescaling of the SOM as in (Cempel and Tabaszewski, 2007), even that generalized fault can be stable and ready for making a diagnostic decision. It is also easy to decide there that the symptom which is primary 6 does not give essential diagnostic information, which can be noticed both as a low contribution in the middle right and as a small weight in the bottom-right panel. A similar decision can be prompted strongly when analyzing the upper panel of Fig. 6, which presents the sum of the absolute weights of columns of the $V$-aligner matrix for the case of a transformed and an untransformed SOM (the transformed case has additionally the system life $\theta$ as the first primary symptom).

The bottom panel of Fig. 6 illustrates the neuron summation concept according to the relation (9). Note that when comparing this panel with the bottom one of Fig. 5, we can notice the same diagnostic information, although they are calculated according to different formulae. This sums of weight as in (9) seems to be a good starting point to connect SVD with neural nets (Dunham, 2003) or some neurons, having the final goal of the prognosis and condition recognition. But this can be the topic of deliberations and calculations, and perhaps a next paper if some advantage will be produced in this way.

As is commonly known, the main goal of condition monitoring is to stop a machine for a renewal before its
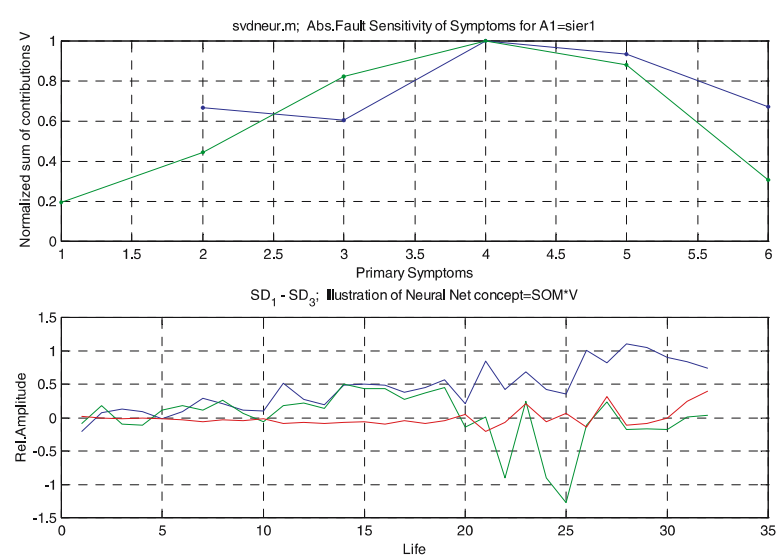

Fig. 6. Sensitivity measure of primary symptoms (top), and an attempt to validate the $\mathrm{NN}$ concept for the fan sier1.

breakdown. So, we should determine the symptom limit value $S_{l}$ which enables us to do this safely. This limit value can base on some experimental practice, some standards (e.g., ISO), or it can be assessed by the new concept of symptom reliability $R(S)$ (Natke, 1997; Cempel, 2000). Figure 7 shows this possibility of assessing the symptom limit value $S_{l}$ (the bottom-right panel), combined with another possibility of optimizing the dimension of the primary symptom observation space. One can notice that Symptom 13 does not give any contribution to the main generalized fault symptoms $1-3$ shown there. Hence 

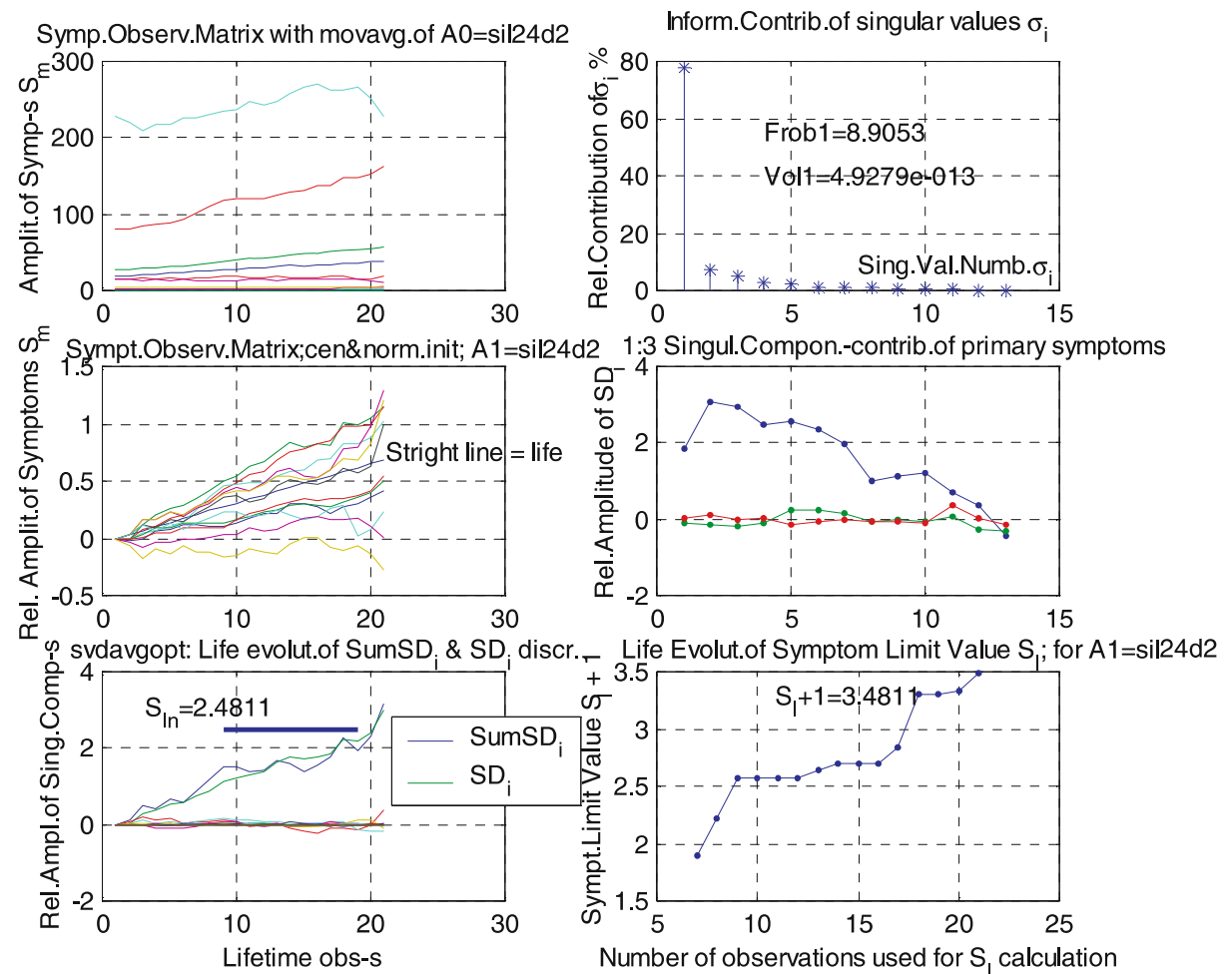

Fig. 7. Diesel engine similar to that considered at the beginning (Figs. 1 and 2) but processed using new multidimensional condition monitoring software assessing the symptom limit value $S_{l}$ and optimizing the dimension of the symptom observation space (one symptom removed).

with any loss of vital information, this primary symptom can be omitted from processing, and this decision can even be made in an automatic way, when this manner of multidimensional data fusion is implemented in some real condition monitoring system.

\section{Condition assessment and forecasting in a multidimensional case}

To the best of the author's knowledge, much is known on the subject of condition assessment of machines and its forecasting in the one-dimensional case, i.e., the case of one fault and one symptom. We can begin this subject by studying some standards, national or international, like ISO2372, looking for a gap between the ISO limit and the measured symptom. The same can be done with respect to the forecasted symptom value. We can use the oldest and simplest method described in (Cempel, 1987) or, e.g., advanced forecasting techniques based on neural nets (Tabaszewski, 2006). However, in our case we have multidimensional observations of machine conditions, and the possibility to extract generalized faults of the object under investigation, as is shown in the bottom panels of Figs. 1 and 7. Here we have the choice to follow each particular generalized fault $\left(S D_{i}\right)$, or to take into consi- deration the total damage advancement in the generalized symptom denoted here as $S u m S D_{i}$. As the generalized fault extracted by SVD is dimensionless in nature and is the result of some linear weighing as explained in Fig. 6, no symptom limit value taken from some standards (e.g., ISO) can be applied in our case. We have to elaborate a special technique of condition assessment and forecasting specific to our multidimensional case. The bottom panels of Fig. 7 are the first step in this direction. Here we have the possibility to predict the system life based on symptom reliability $S_{l}$ calculated in the bottom-right panel. This limit value (denoted here as $S_{l n}$ ) is shown in the bottomleft panel as it determines the system residual life by the cross-section with the generalized total damage symptom SumSDi. This is a direct possibility of system residual life assessment, without a forecast of a next symptom value, SumSDi provided for the generalized total damage symptom in this case.

Concerning symptom value forecasting, there exist now many methods and techniques of forecasting symptom values and conditions (Tabaszewski, 2006), but it seems to be worthwhile to present here a relatively new concept of forecasting flowing directly from grey system theory (GST). This theory, presented for the first time in 1982 by Deng (Deng, 1982; Deng 1989), stems from the idea 
that not all observations of a dynamical system are accurate and reliable enough, partly known, partly unknown, and there must be some inherent mechanisms in the forecasting method to support the inaccurate data of the evolution of the observable system. The area of application covered by GST includes grey relational analysis, grey modelling and prediction, as well as decision making and control. It covers not only all branches of engineering, but economic and social sciences as well. There is no room here to present details of the grey system (GS) prediction methodology. We will describe it qualitatively, passing only some important reference (Wen, 2005).

In general, GST assumes that our incomplete and uncertain observation can be the output of some dynamic multi-input system of a high order described by a grey differential or difference model (Deng, 1982; Wen, 2005). In condition monitoring, we may assume it is a first-order system described by a grey differential equation and one forcing or control input. This simplest case in GST, denoted by $\operatorname{GM}(1,1)$, means the grey model of order 1 with one input only. The output of the system is a series of discrete observations (symptom readings) denoted here as

$$
x^{(0)}=x^{(0)}(1), x^{(0)}(2), \ldots, x^{(0)}(n),
$$

where $n \geq 4$ is the number of observations made on a system, sometimes enough for prediction.

The application of GST to the above symptom readings offers a possibility to forecast the future symptom value, starting from a very small observation number, and using the formula

$$
\hat{x}^{(0)}(k+1)=\left[x^{(0)}(1)-\frac{u}{a}\right]\left(e^{-a k}-e^{-a(k-1)}\right),
$$

$k=2,3, \ldots, n$, where $u$ and $a$ are parameters to be estimated by a special least-squares matrix procedure using the observed data (10). The hat symbol means a forecasted quantity.

As is well known, one of the indicators of forecasting quality is the error of prognosis and the flexibility to follow the symptom evolution. What is more, these two criteria are often contradictory. On the other hand, it has been found in many papers using GST that this error can be much smaller if we use only a small portion of fresh observations, disregarding the old ones. This technique is known as the rolling modelling (Yao, 2004), or one can say that we define and use a forecasting window which remembers only some portion of fresh data.

This forecasting technique was used in our case of multidimensional condition monitoring, and a special Matlab program was prepared to forecast the future values of generalized symptoms of the total damage $S u m S D_{i}$ (see, e.g., Fig. 7) using the grey model $\operatorname{GM}(1,1)$ and the window forecasting technique (rolling modelling). The details of rolling forecasting when applied to condition monitoring are presented in (Cempel et al., 2007). Here we will show only the results and conclusions coming from such symptom processing. Hence, Fig. 7 gives a summary of the grey system forecasting method for the total damage symptom $S u m S D_{i}$ of the engine si124.d1 illustrated already in the bottom-left panel of Fig. 1. The upper panel of Fig. 8 presents the symptom forecast made by the model $\operatorname{GM}(1,1)$ and calculated for the all accessible data, thus without the rolling window. As can be seen, the average forecasting error is not large, but the forecast does not follow the evolution of the symptom, and, what is more, the next forecasted value is almost equal to the previous one observed. However, if we introduce the rolling window (middle panel), the forecast quality improves much with respect to its flexibility, to follow symptom evolution well and diminish the average error of prediction. The last conclusion is shown separately in the bottom panel of Fig. 8, where the average forecasting error is shown as a function of the span of the rolling window. We can observe here that the window span applied, $w=5$, seems to be optimal with respect to the average error as well as the forecasting flexibility.
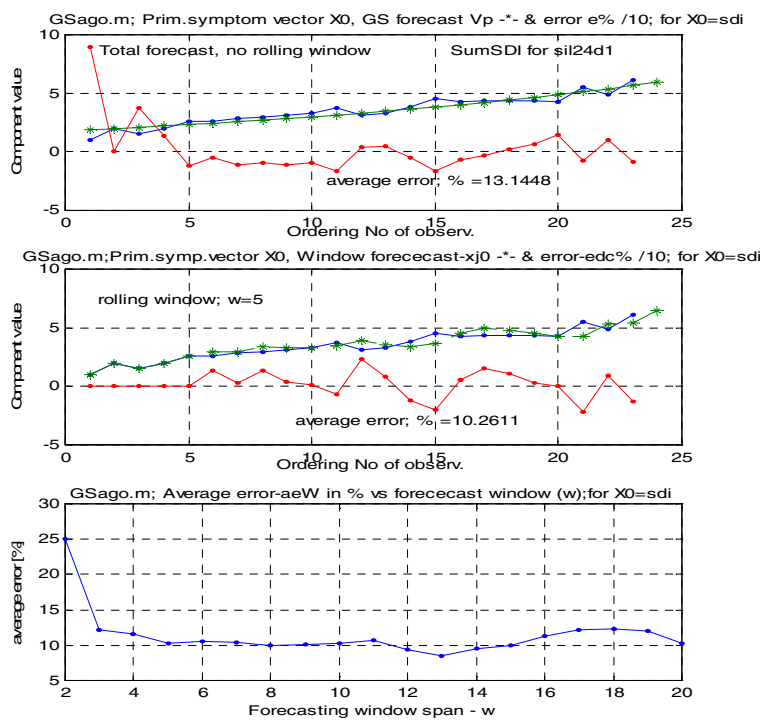

Fig. 8. Specifics of grey system forecasting by a GM(1,1) model, as applied to the generalized symptom $\operatorname{SumSD}_{i}$ of Fig. 1.

If we accept this forecast, we can next compare the forecasted symptom value with the symptom limit valeu $S_{l}$ in a given case. We can find this value processing the data of the sil24d1 engine by the program svdavgopt.m obtaining the limit value as $S_{l}=4.9$. This means that the last three forecasts and two symptom readings already exceeded the calculated symptom limit value. In addition, this seems to be true, since after the last symptom reading the engine crankshaft was broken down. 


\section{Conclusions and further problems}

Looking back at the problems considered and illustrated above as well as in previous papers, we can draw several conclusions and indicate future problems:

- Singular value decomposition (SVD) seems to be a good technique of diagnostic information extraction from the SOM, which is equivalent to, or sometimes better than, principal components analysis (PCA).

- By this decomposition, we can transform the observation space described by the SOM into the needed less dimensional fault space of the observed system. Moreover, we can optimize our observation space, omitting some less fault sensitive primary symptoms.

- Real machine condition monitoring data are sometimes unstable and may have a stochastic component, so rescaling and averaging the symptoms give us more inference power and stable generalized fault symptoms $S D_{i}$.

- There is some analogy of the final step of SVD in calculating the generalized fault symptom (4) to the activity of the set of neurons (9), and this needs more attention and consideration.

- The results of the SVD procedure enable us to determine the symptom limit value $S_{l}$, the quantity most important in vibration condition monitoring.

- Having extracted generalized fault symptoms by SVD, we can pass to the condition recognition and forecasting task, using the calculated symptom limit value $S_{l}$.

- For the forecasting task, the use of grey system theory was shown here, which is particularly suited for short data sequences which are sometimes encountered in the machine condition monitoring area. This technique gives us good a flexibility of forecast and a small average error.

\section{References}

Bartelmus W., Zimroz Z. and Batra H. (2003). Gearbox vibration signal preprocessing and input values choice for neural network training, Proceedings of the Conference on AI Methods, Gliwice, Poland.

Cempel C. (1999). Innovative developments in systems condition monitoring, Keynote Lecture, Proceedings of the Conference on Damage Assessment DAMAS'99, Dublin, Ireland, pp. 172-188.

Cempel C., Natke H.G. and Yao J.P.T. (2000). Symptom reliability and hazard for systems condition monitoring, Mechanical Systems and Signal Processing 14(3): 495-505.

Cempel C. (2003). Multidimensional condition monitoring of mechanical systems in operation, Mechanical Systems and Signal Processing 17(6): 1291-1303.
Cempel C. and Tabaszewski M. (2007). Multidimensional vibration condition monitoring of nonstationary systems in operation, Mechanical Systems and Signal Processing 21(3): 1233-1241.

Cempel C., Krakowiak, M. (2006a). Influence of running stability and randomness of observation on the condition assessment in multidimensional machine diagnostics, Diagnostyka 40(4): 19-25 .

Cempel C. and Tabaszewski M. (2006b). Averaging the symptoms in multidimensional condition monitoring for machines in nonstationary operation, Proceedings of the $13 \mathrm{In}$ ternational Congress on Sound and Vibration, Vienna, Austria, CD-ROM.

Cempel C. (2004). Implementing multidimensional inference capability in vibration condition monitoring, Proceedings of the Conference on Acoustical and Vibratory Surveillance, Senlis, France.

Cempel C. (2005). Multi fault vibrational diagnostics of critical machines, Zagadnienia Eksploatacji Maszyn 4(144): 133 142.

Cempel C. (1991). Vibroacoustic Condition Monitoring, Ellis Horwood, London.

Cempel C. (1987). Simple condition forecasting techniques in vibroacoustical diagnostics, Mechanical Systems and $\mathrm{Si}$ gnal Processing 1(1): 75-82.

Cempel C. (2008). Forecasting the global and partial system condition by means of multidimensional condition monitoring, Journal of Theoretical and Applied Mechanics 46(4): 777797

Deng J.L. (1982). Control problems of grey systems, Systems and Control Letters 1(5): 288-294.

Deng J-L. (1989). Introduction to grey system theory, The Journal of Grey Systems 1(1): 1-24.

Dunham M.H. (2003). Data Mining_Introductory and Advanced Topics, Prentice Hall, Englewood Cliffs, NJ.

Golub G.H., VanLoan C. F. (1983), Matrix Computation, North Oxford Academic, Oxford.

Jasiński M. (2004). Empirical models in gearbox diagnostics, Ph.D. thesis, Warsaw University of Technology, (in Polish).

Korbicz J., Koscielny J.M., Kowalczuk Z. and Cholewa W. (Eds.) (2004). Fault Diagnosis-Models, Artificial Intelligence, Applications, Springer Verlag, Berlin.

Kiełbasiński A. and Schwietlick H. (1992). Numeric Linear Algebra, WNT, Warsaw, (in Polish).

Natke H. G. and Cempel C. (2002). The symptom observation matrix for monitoring and diagnosis, Journal of Sound and Vibration 248(4): 597-620.

Natke H. G. and Cempel C. (1997). Model Aided Diagnosis of Mechanical Systems, Springer-Verlag, Berlin.

Pantopian N. H. and Larsen J. (1999). Unsupervised condition detection in large diesel engines, Proceedings of the IEEE Workshop on Neural Networks. 
Tabaszewski M. (2006). Forecasting of residual life of a fan mill by means of neural nets, Diagnostyka 3(39): 149-156, (in Polish).

Tumer I.Y. and Huff E.M. (2002). Principal component analysis of tri-axial vibration data from helicopter transmission, Proceedings of 56th Meeting of the Society of Machine Failure Prevention Technology.

Wen K.L. and Chang T. C. (2005). The research and development of completed $\mathrm{GM}(1,1)$ model toolbox using Matlab, International Journal of Computational Cognition 3(3): 42-48.

Will T. (2005). Hanger matrix, two-thirds theorem, available at: http: / /www. uwlax.edu/faculty/will/ svd/svd/index.html.
Żółtowski B. and Cempel C. (Eds.) (2004). Engineering of Machine Diagnostics, ITE Press, Radom, p. 1308, (in Polish).

Yao A.W.L. and Chi S.C. (2004). Analysis and design of a Taguchi-Grey based electricity demand predictor for energy management systems, Energy Conversion and $M a$ nagement 45: 1205-1217.

Received: 4 October 2007 Revised: 11 April 2008 
